\title{
Optimization of two GPS/MEMS-IMU integration strategies with application to sports
}

\author{
Adrian Waegli · Jan Skaloud
}

Received: 4 September 2008/ Accepted: 10 March 2009

(C) Springer-Verlag 2009

\begin{abstract}
The application of low-cost L1 GPS receivers integrated with micro-electro-mechanical system (MEMS) inertial measurement units (IMU) allows the continuous observation of position, velocity and orientation which opens new possibilities for comparison of athletes' performance throughout a racecourse. In this paper, we compare loosely and closely coupled integration strategies under realistic racing scenarios when GPS is partially or completely masked. The study reveals that both integration approaches have a similar performance when the satellite constellation is completed or the outages are short. However, for less than four satellites, the closely coupled strategy clearly outperforms the loosely coupled approach. The second part of the paper is devoted to the important problem of system initialization, because the conventional GPS/IMU alignment methods are no longer applicable when using MEMS-IMU. We introduce a modified coarse alignment method and a quaternion estimation method for the computation of the initial orientation. Simulations and practical experiments reveal that both methods are numerically stable for any initial orientation of the sensors with the error characteristics of MEMS-IMUs. Throughout the paper, our findings are supported by racing experiments with references provided in both, the measurement and the navigation domains.
\end{abstract}

\footnotetext{
A. Waegli $(\square) \cdot$ J. Skaloud

Ecole polytechnique fédérale de Lausanne (EPFL),

Lausanne, Switzerland

e-mail: adrian.waegli@epfl.ch

URL: http://topo.epfl.ch/

J. Skaloud

e-mail: jan.skaloud@epfl.ch
}

Keywords GPS/INS integration · MEMS-IMU · MEMS magnetometer · Navigation performance . Loosely and closely coupled integration . Orientation initialization · Sport

\section{Introduction}

Every discipline of sports has its own techniques for performance analysis. The requirements for such methods depend on the discipline's motion, ergonomic constraints and cost related to the discipline. For disciplines implying fast body motion of longer duration, the investigation of the trajectory is of utmost importance.

\section{Performance criteria in sports}

Athletes and coaches are not only interested in the positions and velocities, but also in the motion analysis of segments of the human or the orientation of equipment (Waegli et al. 2007a, 2008). Furthermore, the knowledge of position serves as a base for comparing other performance criteria (e.g. heart rates, rotations per minute) between concurrent or on repeated runs (Waegli 2009).

Table 1 summarizes the requirements of sport performance evaluation techniques in terms of timing, position, velocity, and orientation accuracies. There, three categories are identified that possess distinct performance criteria: Trajectory comparison, material testing and development, as well as motion analysis and rehabilitation. Our experience shows that sport professionals tend to overestimate the need in position accuracy. The centimeter to decimeter level positioning is crucial only for a few applications, namely, those related to timing (material testing) and disciplines where small trajectory differences can be 
Table 1 Accuracy requirements for trajectory determination in sports

\begin{tabular}{|c|c|c|c|c|c|c|c|}
\hline Field of use & $\begin{array}{l}\text { Timing } \\
\text { (s) }\end{array}$ & Position & $\begin{array}{l}\text { Velocity } \\
(\mathrm{km} / \mathrm{h})\end{array}$ & $\begin{array}{l}\text { Acceleration } \\
(\mathrm{g})\end{array}$ & $\begin{array}{l}\text { Orientation } \\
(\text { deg })\end{array}$ & $\begin{array}{l}\text { Sampling } \\
\text { frequency } \\
(\mathrm{Hz})\end{array}$ & Remarks \\
\hline \multicolumn{8}{|l|}{ Trajectory comparasion } \\
\hline Post-mission & & $<0.5 \mathrm{~m}$ & $<1$ & & $1-2$ & $1-5$ & $\begin{array}{l}\text { Few application require dm- } \\
\text { accuracy (e.g. slalom in skiing), } \\
\text { relative position acuracy is more } \\
\text { important than absolute }\end{array}$ \\
\hline Real-time (e.g. TV broadcasting & & $1-10 \mathrm{~m}$ & $1-2$ & \pm 1 & & & $\begin{array}{l}\text { Includes trajectory matching and } \\
\text { comparison algorithms }\end{array}$ \\
\hline Chronometry & $1 / 100$ & $\begin{array}{l}20 \mathrm{~cm} \text { at } \\
80 \mathrm{~km} / \mathrm{h}\end{array}$ & $<1$ & & & Max. 5 & $\begin{array}{l}\text { GPS or GPS/INS derived } \\
\text { chronometry depends on the } \\
\text { athlete's velocity and the } \\
\text { position accuracy }\end{array}$ \\
\hline \multicolumn{8}{|l|}{ Material testing and developed } \\
\hline Tire development, ski testing & & $<0.2 \mathrm{~m}$ & $1-2$ & & $1-2$ & 1 & $\begin{array}{l}\text { Positin and velocity of the ski are } \\
\text { required, no crossing of the ski }\end{array}$ \\
\hline Vibrations & & & & & & 400 & $\begin{array}{l}\text { Max. vibrations in motor sports at } \\
200 \mathrm{~Hz} \text {, muscles absorb } \\
\text { vibrations down to } 20 \mathrm{~Hz}\end{array}$ \\
\hline $\begin{array}{l}\text { Gliding and aerodynamic } \\
\text { properities (suit, ski) }\end{array}$ & $1 / 100$ & $\begin{array}{l}20 \mathrm{~cm} \text { at } \\
80 \mathrm{~km} / \mathrm{h}\end{array}$ & $<1$ & & & 1 & $\begin{array}{l}\text { Closely related to the timing } \\
\text { accuracy, trajectory modeling }\end{array}$ \\
\hline \multicolumn{8}{|l|}{ Motion analysis and rehabilitation } \\
\hline Evaluation of the athlete's motion & & & & & 2 & 50 & Orientation of the body segments \\
\hline Energy transfer & & CG: $10 \mathrm{~cm}$ & CG: $1-2$ & & & 50 & $\begin{array}{l}\text { Qualitatively accurate data more } \\
\text { important than quantitatively }\end{array}$ \\
\hline
\end{tabular}

important (e.g. slalom in Alpine skiing). Secondly, there is often confusion about the needs in terms of absolute and relative accuracies. For instance, in many disciplines the trajectory shape (meaning high relative accuracy) is of greater importance than the absolute positioning accuracy. This is true also for energy transfer computations where sports professionals are more interested in relative changes rather than absolute values.

Extension of the limits in satellite-based positioning

Satellite-based positioning fulfills some of the criteria presented in Table 1 and has already proven its effectiveness in car racing (How et al. 2002), rowing (Zhang et al. 2003, 2004) and Alpine skiing (Skaloud and Limpach 2003). However, the athlete's environment is quickly alternating between open space and adverse areas to the reception of satellite signals (sudden satellite obstruction). Therefore, it is difficult or even impossible to resolve the carrier-phase ambiguities. Considering the ergonomic requirements placed on the equipment worn on the body, current technological limits in GPS positioning are quickly reached or even exceeded (Skaloud and Merminod 2000). Furthermore, the use of dual-frequency GPS receivers is reserved for a few sports applications with higher accuracy needs because of ergonomic and economic restrictions. In addition, many sports professionals are not only interested in position and velocity but also in orientation. For these reasons, we introduced a system that integrates low-cost L1 GPS receivers with low-cost micro-electro-mechanical system (MEMS) inertial measurement units (IMU) (Waegli and Skaloud 2007). The MEMS device that is suitable in size and cost consists of inertial sensors (triple axis accelerometer and gyroscopes), as well as a triad of magnetometers. The principal benefit of this combination is in overcoming the lack of continuity of the GPS signals in a difficult environment and determining the body orientation accurately $(<1 \mathrm{deg})$.

\section{Previous experiences}

In a study (Waegli and Skaloud 2007), we have shown that an extended Kalman smoother implemented for post-processing and in loosely coupled approach was able to bridge GPS gaps of a duration of less than $10 \mathrm{~s}$ without significant degradation of positioning accuracy. Closely coupled methods allow integrating GPS and inertial measurements even if the number of observed satellites drops below four (Wei and Schwarz 1990; Sukkarieh and George 2005). In this approach, GPS data from individual satellites can be used or rejected at the measurement update of the Kalman filter. According to (Schwarz et al. 1994; Wendel et al. 
2005), the closely coupled approach is more robust for an incomplete constellation and it offers superior performance as compared to loosely coupled systems under these circumstances. It is reported that for very poor geometry (PDOP $>50$ ), the closely coupled approach presents some advantages. When the number of satellites falls below three, the positioning accuracy deteriorates rapidly and is dominated by the errors of inertial navigation. Sukkarieh and George (2005) have shown in simulations that for partial GPS outages lasting $20 \mathrm{~s}$, the horizontal position error was improved by a factor of 15 where only two GPS satellites were visible. However, where the satellite geometry was reasonable (i.e. more than four visible satellites), the closely coupled approach did not present significant improvement over the loosely coupled (Schwarz et al. 1994). Previous research also reported comparable performance navigation accuracy for dynamics typical of sports using sigma-point Kalman filter (SPKF). There, loosely (Gelb 1994) and closely (Wendel et al. 2005) coupled integration yielded MEMS-IMU calibration with similar accuracies. As the performance is comparable and implies higher computational cost, the presented investigation employs only EKF-based filtering.

Outline of the article

In this paper, we first present the theoretical background related to the loosely and closely coupled integration strategies. Then, the latter are assessed based on an experiment where a professional downhill skier was equipped with single and dual-frequency GPS, MEMSinertial and magnetic sensors and a tactical-grade inertial unit serving as a reference. The study is refined by resampling GPS data in order to simulate reduced satellite constellations over specified time intervals.

The second part of this article focuses on the initial alignment which is a critical component in GPS/MEMSIMU integration. We introduce and compare two methods for the initialization of the orientation. The first algorithm is a modified coarse alignment approach where the gyroscope measurements are replaced by magnetic measurements. The second method is a quaternion-based approach using accelerometer and magnetometer observations. The limitations of both algorithms with respect to the MEMS sensor error characteristics are presented.

\section{Integration strategy}

IMU strapdown inertial navigation

Expressing the strapdown differential equations in the local level frame (index $n$ ) makes the interpretation of the navigation state variables straightforward (Titterton and Weston 1997). The trajectory is parameterized by the position $\mathbf{r}$ and velocity vectors $\mathbf{v}$ as well as the rotation matrix $\mathbf{R}_{b}^{n}$. Supposing that the initial values of these parameters are known, their evolution in time can be derived with $\boldsymbol{\omega}_{\mathrm{ib}}^{b}$ and $\mathbf{f}^{b}$, the gyroscope and accelerometer measurements, based on the following relations:

$$
\begin{aligned}
\dot{\mathbf{r}}^{n} & =\mathbf{D}^{-1} \mathbf{v}^{n} \\
\dot{\mathbf{v}}^{n} & =\mathbf{R}_{b}^{n} \mathrm{f}^{b}-\left(2 \boldsymbol{\omega}_{\mathrm{ie}}^{n}+\boldsymbol{\omega}_{\mathrm{en}}^{n}\right) \times \mathbf{v}^{n}+\mathbf{g}^{n} \\
\dot{\mathbf{R}}_{b}^{n} & =\mathbf{R}_{b}^{n} \mathbf{\Omega}_{\mathrm{nb}}^{b}
\end{aligned}
$$

where

$\mathbf{D}^{-1}=\left[\begin{array}{ccc}\frac{1}{M+h} & 0 & 0 \\ 0 & \frac{1}{(N+h) \cdot \cos \varphi} & 0 \\ 0 & 0 & -1\end{array}\right]$

and

$\boldsymbol{\omega}_{\mathrm{nb}}^{n}=\boldsymbol{\omega}_{\mathrm{ib}}^{b}-\mathbf{R}_{b}^{n}\left(\boldsymbol{\omega}_{\mathrm{ie}}^{n}+\boldsymbol{\omega}_{\mathrm{en}}^{n}\right)$

where $M$ and $N$ are the principle ellipsoidal radii of curvature, $h$ the altitude and $\varphi$ the geographic latitude. $\omega_{\mathrm{ie}}^{n}$ is the rotation rate of the Earth expressed in the navigation frame, $\omega_{\mathrm{en}}^{n}$ the rotation rate of the local geographic frame with respect to the Earth fixed frame. $\boldsymbol{\Omega}_{\mathrm{nb}}^{b}$ is the skewsymmetric form of $\omega_{\mathrm{nb}}^{n}$ which is the rotation rate of the body frame with respect to the navigation frame. $\mathbf{g}^{n}$ stands for the normal gravity.

For the inertial measurements, a simplified error model was considered. Previous research presented in Waegli et al. (2007b) revealed that misalignments, drifts and constant offsets cannot be decorrelated efficiently given the characteristics of the MEMS sensors and limited integration periods. Therefore, only bias terms are taken into account and modeled as first order Gauss-Markov processes:

$$
\begin{aligned}
& \hat{\ell}^{b}=\ell^{b}+\mathbf{b}_{\ell^{b}}+\mathbf{w}_{\ell^{b}} \\
& \dot{\mathbf{b}}_{\ell^{b}}=-\beta_{\ell^{b}} \mathbf{b}_{\ell^{b}}+\sqrt{2 \sigma_{\ell^{b}}^{2} \beta_{\ell^{b}}} \mathbf{w}_{\ell^{b}}
\end{aligned}
$$

where $\hat{\ell}^{b}$ is the estimated inertial observation (specific force or rotation rate), $\ell^{b}$ the inertial measurement, $\mathbf{b}_{\ell^{b}}$ the bias of the inertial measurement, $\mathbf{w}_{\ell^{b}}$ the measurement noise, $\sigma_{\ell^{b}}^{2}$ the amplitude of the power spectral density and $\beta_{\ell^{b}}$ the inverse of the correlation time (Gelb 1994).

Loosely coupled GPS updates

In the loosely coupled approach, GPS coordinates and velocities are fed to a filter (e.g. an extended Kalman filter EKF) as measurement updates. Accounting for the leverarm effect, the GPS position measurement model can be expressed as follows: 
$\mathbf{r}_{\mathrm{GPS}}^{n}=\mathbf{r}_{\mathrm{IMU}}^{n}+\mathbf{D}^{-1} \mathbf{R}_{b}^{n} \mathbf{a}^{b}+\mathbf{w}_{r}$

where $\mathbf{a}^{b}$ is the lever-arm vector between the INS center of navigation and the GPS antenna phase center and $\mathbf{w}_{r}$ the residual position error.

The GPS velocity measurement model needs to consider the rotation of the local level frame with respect to the body frame $\omega_{\mathrm{nb}}^{n}$ :

$\mathbf{v}_{\mathrm{GPS}}^{n}=\mathbf{v}_{\mathrm{IMU}}^{n}+\boldsymbol{\omega}_{\mathrm{nb}}^{n} \times \mathbf{R}_{b}^{n} \mathbf{a}^{b}+\mathbf{w}_{v}$

The MEMS magnetometers can be introduced as external measurements as well. However, Waegli and Skaloud (2007) and Törnqvist (2006) have pointed out that the performance of these sensors was sensitive to high accelerations. Waegli and Skaloud (2007) further pointed out that the use of magnetic measurements improved the orientation estimation only negligibly and thus it does not affect the capacity of the MEMS sensors to bridge GPS gaps. Therefore, the magnetometers are not applied in this investigation except during the phase of sensor initialization.

\section{Closely coupled GPS updates}

In the closely coupled approach, GPS observations (carrierphase smoothed pseudoranges and Doppler measurements) are fed to the EKF at the update stage. Unlike in the loosely coupled approach, GPS measurements are processed also if the GPS receiver does not provide a position fix as is the case when less than $4 \mathrm{SVs}$ are available. In our implementation, double-differenced (DD) GPS measurements are applied which eliminates satellite and receiver clock errors and mitigates atmospheric errors. The DD pseudorange $\nabla \Delta p_{m, r}^{z, i}$ between an arbitrary satellite $i$, reference satellite $z$, rover GPS antenna $r$ and master GPS antenna $m$ can be expressed as follows:

$\nabla \Delta p_{m, r}^{z, i}=\nabla \Delta \rho_{m, r}^{z, i-}+\nabla \Delta M_{m, r}^{z, i}+w_{p}$

where DD operator $\nabla \Delta$ of quantity $x$ is defined as

$\nabla \Delta x=\left(x_{m}^{z-}-x_{r}^{z-}\right)-\left(x_{m}^{i-}-x_{r}^{i-}\right)$

and the approximated range $\rho_{r}^{i}$ is calculated as: complex multipath mitigation methods can be found in the literature (Van Dierendonck and Braasch 1997; Ge et al. 2000; Braasch 2001; Yang et al. 2004). However, their evaluation is beyond the scope of this research. The short baselines, as encountered in many sport applications, do not require more accurate modeling of the errors affecting GPS (ionosphere, troposphere). Using only L1 measurements, we do not attempt to solve ambiguities but simply smooth the pseudoranges by the carrier-phase measurements (Hatch 1982):

$p_{s, k}=\frac{1}{W} p_{k}+\frac{W-1}{W}\left(p_{s, k-1}+\left(\phi_{k}-\phi_{k-1}\right)\right)$

where $p_{s, k}$ is the smoothed, $p_{k}$ the raw pseudorange observation and $\phi_{k}$ the carrier-phase measurement at epoch $k$. $W$ stands for the smoothing window duration [usually set to 100 epochs (Hofmann-Wellenhof et al. 1992)]. The double-differenced Doppler measurements are derived as follows :

$\nabla \Delta \dot{p}_{m, r}^{z, i}=\nabla \Delta \dot{\rho}_{m, r}^{z, i-}+w_{\dot{p}}$

with

$\dot{\rho}_{r}^{i-}=\left(\mathbf{v}^{i}-\mathbf{v}_{r}\right)^{T}\left(\frac{\mathbf{r}^{i}-\mathbf{r}_{r}}{\left\|\mathbf{r}^{i}-\mathbf{r}_{r}\right\|}\right)$

where $\dot{p}$ is the pseudorange rate observation and $\dot{\rho}$ the true pseudorange rate.

\section{Trajectory smoothing}

The visibility of satellites often varies along the tracks. By post-processing the trajectories in forward and backward direction, the solution during periods with weak satellite constellations can be improved. The following fixedinterval smoothing algorithm combines forward- and backward-filtered data sets in the least squares sense (Meditch 1969; Shin 2005):

$$
\begin{aligned}
& \mathbf{x}_{s}=\mathbf{x}_{f}^{+}+\mathbf{P}_{s} \mathbf{P}_{b}^{-1}\left(\mathbf{x}_{b}^{+}-\mathbf{x}_{f}^{+}\right) \\
& \mathbf{P}_{s}=\left(\mathbf{P}_{f}^{-1}+\mathbf{P}_{b}^{-1}\right)^{-1}
\end{aligned}
$$

$\rho_{r}^{i-}=\sqrt{\left(g\left(\mathbf{r}^{n, i}\right)-g\left(\mathbf{r}_{\mathrm{IMU}}^{n}+\mathbf{D}^{-1} \mathbf{R}_{b}^{n} \mathbf{a}^{b}\right)\right)^{T} \cdot\left(g\left(\mathbf{r}^{n, i}\right)-g\left(\mathbf{r}_{\mathrm{IMU}}^{n}+\mathbf{D}^{-1} \mathbf{R}_{b}^{n} \mathbf{a}^{b}\right)\right)}+c \cdot \delta t$

with $g$ representing the transformation function from curvilinear to Cartesian coordinates. The multipath $M$ can be identified by evaluating the difference $p_{r}^{i-}-\rho_{r}^{i}$. If this is the case, the affected observation can be removed. More where $\mathbf{x}$ is the predicted (superscript - ) or adjusted (superscript + ) state vector with corresponding covariance matrix $\mathbf{P}$. Indexes $f, b$ and $s$ denote the forward or backward processed and the smoothed states or covariances. 


\section{System initialization}

Position and velocity are initialized by GPS measurements. Alternatively, if the athlete is not moving, the initial coordinates can be introduces manually and the velocity set to zero. The determination of the initial orientation is more complicated. The coarse alignment method applied in conventional GPS/INS integration considers two pairs of vectors which are both available in the local level frame $n$, which is also the navigation frame, and the body frame $b$. The local level projection of these two vectors is known from the model (Earth gravity and rotation) while the body frame projections are obtained from the sensor measurements (specific force and angular rate). Unfortunately, the error characteristics of the MEMS gyroscopes (noise level $>0.1 \mathrm{deg} / \mathrm{s} / \mathrm{sqrt}(\mathrm{Hz})$, systematic errors of several deg/s) do not allow sensing the Earth's rotation rate. Hence, this conventional alignment method cannot be employed. Therefore, two alternative approaches exploiting MEMS magnetometers are envisaged: a modified coarse alignment and a quaternion-based algorithm. Both will be presented in the sequel.

\section{Modified coarse alignment method}

The MEMS magnetic measurements and the a priori known magnetic field of the Earth offer an alternative pair of vectors that replace the gyroscope measurements. Thus, the orientation of a rigid body $\mathbf{R}_{n}^{b}$ can be determined from the following relation:

$\left[\begin{array}{lll}-\mathbf{f}^{b} & \mathbf{m}^{b} & -\mathbf{f}^{b} \times \mathbf{m}^{b}\end{array}\right]=\mathbf{R}_{n}^{b}\left[\begin{array}{lll}\overline{\mathbf{g}}^{n} & \mathbf{m}^{n} & \overline{\mathbf{g}}^{n} \times \mathbf{m}^{n}\end{array}\right]$

where $\mathbf{f}^{b}$ is the specific force measurement, $\overline{\mathbf{g}}^{n}$ the normal gravity vector, $\mathbf{m}^{b}$ the magnetic observation and $\mathbf{m}^{n}$ the Earth magnetic field deduced from any global or local reference model [e.g. the World magnetic model (WMM) provided by the National Geographic Data Center, http://www.ngdc.noaa.gov/geomag]. $\mathbf{f}^{b}$ and $\mathbf{m}^{b}$ can be averaged over a period during which the sensor does not move. In the coarse alignment algorithm the rotation matrix must be converted to the quaternion form. This step is potentially unstable. Indeed, in the cases where the trace of the rotation matrix equals -1 and the off-diagonal terms are skew-symmetric, a zero quaternion is generated.

\section{QUEST algorithm}

Exploiting again the Earth's gravity and magnetic fields, the initial orientation can also be sought based on weighted (w) observations and their external reference expressed as unit vectors [Wahba's problem (Wahba 1965)].
$J(\mathbf{q})=\frac{1}{m} \sum_{i=1}^{m} w_{k}\left(\mathbf{b}_{i}-\mathbf{R}_{n}^{b} \mathbf{n}_{i}\right)^{2}$

where $J$ is a cost function with, the number of employed sensors (magnetometers and accelerometers). $\mathbf{b}_{i}$ are the measurement in the body frame (the specific force measurement $\mathbf{f}^{b}$ and the magnetic observation $\mathbf{m}^{b}$ ) and $\mathbf{n}_{i}$ the reference values (the normal gravity vector $\overline{\mathbf{g}}^{n}$ and the Earth magnetic field $\mathbf{m}^{n}$ ).

An elegant solution for quaternion based algorithms is referred to as the QUEST (QUaternion ESTimation) algorithm (Schuster and Oh 1981). The attitude can be found unambiguously when minimizing the expression

$J(\mathbf{q})=\frac{1}{2} \sum_{i=1}^{2} w_{k}\left(\mathbf{b}_{i}-\mathbf{R}_{n}^{b} \mathbf{n}_{i}\right)^{2}$

with the condition $\mathbf{q}^{T} \cdot \mathbf{q}=1$. In contrast to the coarse alignment algorithm, the QUEST algorithm directly provides a quaternion which is employed in the strapdown navigation without further transformation.

Adaptation for dynamic initialization

If the initialization is to be performed under dynamic conditions, the specific force measurement needs to be corrected for the kinematic acceleration. This acceleration can be derived from GPS measurements in the navigation frame and fed back to the coarse alignment or to the QUEST algorithm:

$\mathbf{f}^{n}=\mathbf{a}^{n}+\left(\boldsymbol{\omega}_{\text {in }}^{n}+\boldsymbol{\omega}_{\text {ie }}^{n}\right) \times \mathbf{v}^{n}-\mathbf{g}^{n}$

where $\mathbf{a}^{n}$ and $\mathbf{v}^{n}$ are the acceleration and velocity vectors derived from GPS respectively (Bruton et al. 1999; Skaloud and Limpach 2003). Furthermore, the gyroscope measurement can be accounted for between epochs by means of the elegant recursive QUEST (REQUEST) algorithm (Bar-Itzhack 1996; Li et al. 2006).

\section{Experimental setup}

Downhill skiing is an ideal discipline for testing the proposed integration methods because of the important dynamics and because of the difficult satellite reception imposed by the mountainous environments. To investigate the navigation performance of the low-cost L1 GPS/ MEMS-IMU setup, the instruments were mounted in a backpack together with a reference system, comprising a dual-frequency GPS receiver (Javad) and a tactical-grade IMU (LN200). The accuracy of the reference solution is estimated to $5 \mathrm{~cm}$ (position), $2 \mathrm{~cm} / \mathrm{s}$ (velocity), $0.01 \mathrm{deg}$ (roll, pitch) and $0.03 \mathrm{deg}$ (heading). The MEMS sensor 


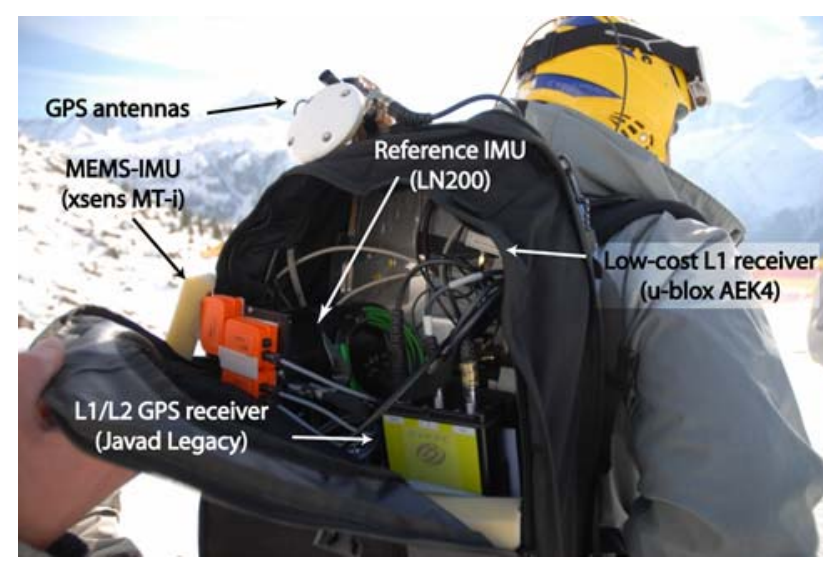

Fig. 1 Experimental setup mounted on a professional skier

(Xsens MTi) was fixed rigidly to the reference IMU with a constant lever-arm (Fig. 1) together with a low-cost u-blox AEK4 GPS receiver. A dozen of downhill runs of approximately $1 \mathrm{~min}$ length each were performed by a professional skier. Each run was preceded by a static initialization phase of $2-3 \mathrm{~min}$.

\section{Assessment of the integration strategy}

The integration strategy is investigated in three steps. Firstly, we compare the accuracy provided by different GPS receivers, e.g. geodetic receivers with fixed/float carrier-phase ambiguities versus low-cost receivers with smoothed carrier-phase measurements. Secondly, we integrated the MEMS-IMU measurements $(100 \mathrm{~Hz})$ with GPS updates $(1 \mathrm{~Hz})$ based on the loosely and closely coupled integration approaches. The performance using L1 measurements of different quality and GPS algorithms is evaluated. In the third step, we refine the assessment of the performance provided by the two integration strategies. In this context, the GPS data sets are resampled by removing satellite measurements over a different time intervals (5-40 s). It is important to stress that an outage of $40 \mathrm{~s}$ corresponds to two-thirds of the run with only $10 \mathrm{~s}$ of satellite data remaining after the start and before the arrival. The SVs with small elevation were removed first, which corresponds to artificially increasing the surrounding topography. All comparisons were performed for six independent downhill runs.

\section{Satellite navigation performance}

The performance of different solutions is assessed with respect to the reference solution provided by the dualfrequency GPS/INS LN200 trajectory processed by commercial software packages (Applanix PosProc v.2.1 and Novatel GrafNav v.7.5). Figure 2 summarizes the root

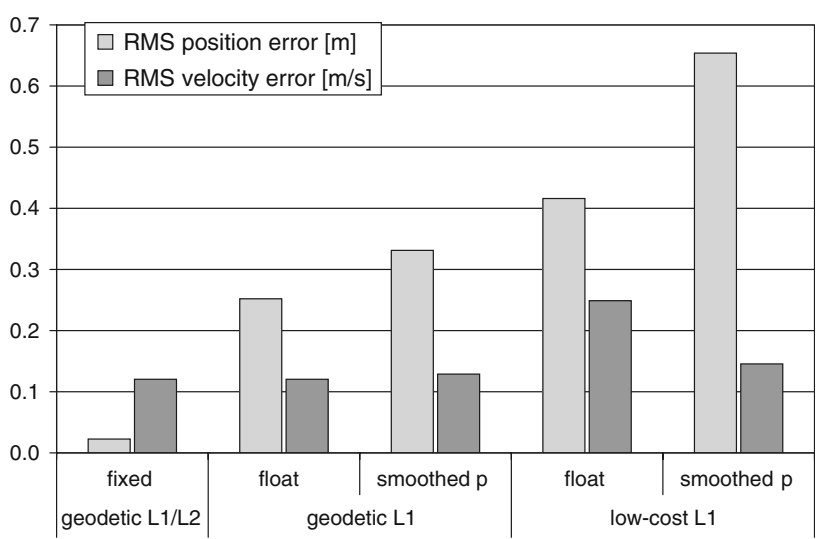

Fig. 2 Position and velocity RMSE of differential GPS solutions for six downhill runs as a function of the receiver hardware (geodetic L1 or L1/L2, low-cost L1 GPS receiver) and the ambiguity resolution (fixed during more than $95 \%$ of time, float, carrier-phase smoothed pseudoranges)

mean square errors (RMSE) of the GPS position and velocities based on different processing and receivers averaged over three axes.

The L1/L2 solution presents an average positional accuracy below decimeter level despite the difficulty in maintaining fixed ambiguities because of the rapidly changing signal reception due to the mountainous environment. Indeed, the ambiguities remain float on certain portions of the track. The position RMSE increases for the single-frequency solutions to a level of $30 \mathrm{~cm}$ using the same receiver and to half-meter level using the low-cost receiver. The accuracy is improved when the carrier-phase smoothing of pseudoranges is replace by float ambiguity estimation (improvement of 30-50\%). Except in the case of the low-cost receiver, there is no significant accuracy difference between the horizontal and vertical components.

In the velocity domain however, the accuracy differences are much smaller between the GPS solutions. Indeed, the velocity computation is independent of the ambiguity resolution and uses the same observations (Doppler measurements). It is more surprising that the quality of the GPS receiver does not significantly improve the velocity estimation. Except the solution with the low-cost receiver and float ambiguities all solutions provide $\mathrm{dm} / \mathrm{s}$ accuracy. The larger velocity error in this case is probably originated by differing filter settings in the GPS processor, i.e. a commercial software (Novatel) where not all parameters can be controlled.

\section{Overall GPS/MEMS-IMU performance}

The L1 GPS observations from geodetic and low-cost receivers were integrated with MEMS-IMU measurements based on the presented loosely and closely coupled 


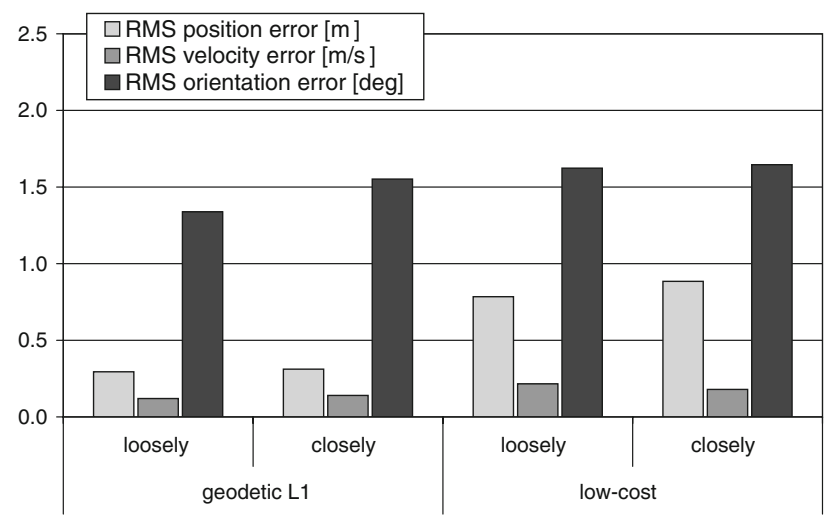

Fig. 3 RMSE for position, velocity and orientation of six runs based on the loosely and the coupled approach with carrier-phase smoothed pseudoranges

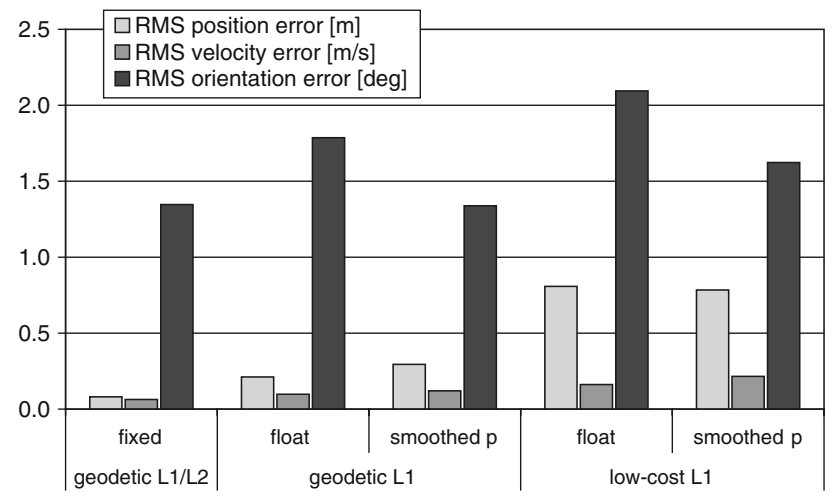

Fig. 4 RMSE for position, velocity and orientation of six runs based on the loosely coupled approach

integration schemes and compared to the reference solution. Figures 3 and 4 summarize the root mean square errors (RMS) of the GPS/INS solutions for the six downhill runs averaged over the three components.

We can observe that the position error is mainly driven by the accuracy of differential GPS (with floating ambiguities or carrier-phase smoothed pseudo-range measurements) and thus corresponds to the values presented in Fig. 2. The integration of the MEMS-IMU measurements does not affect the velocity accuracy. The obtained orientation performance is comparable in all approaches. For all trajectory states, the difference between loosely and closely coupled integrations schemes is negligible. Again, the small discrepancies may be originated by the filter settings in the GPS processing.

GPS/MEMS-IMU performance during reduced satellite reception

In the previous section, we have shown that the loosely and closely coupled approaches performed equally under reasonable satellite constellations ( $>4 \mathrm{SVs})$. However, an accuracy improvement can be expected for the closely coupled strategy under partial satellite constellations (Wei and Schwarz 1990; Schwarz et al. 1994; Sukkarieh and George 2005; Wendel et al. 2005). Such scenarios can be simulated by resampling GPS data sets and removing satellites observations over a variable period of time (5-40 s). In this section, we analyze the two integration strategies under such conditions in terms of position, velocity and orientation accuracies. This evaluation is based on L1 GPS measurements from the geodetic receiver.

The overall accuracy improvement provided by the closely coupled integration approach with respect to the loosely coupled approach is computed for each data set based on

$\mathrm{RMSE}_{\text {improvement }}=\left\{\begin{array}{lll}\left(\frac{\mathrm{RMSE}_{\text {loosely }}}{\mathrm{RMSE}_{\text {closely }}}-1\right) & \text { if } & \frac{\mathrm{RMSE}_{\text {loosely }}}{\mathrm{RMSE}_{\text {closely }}}>1 \\ \left(1-\frac{\mathrm{RMSE}_{\text {closely }}}{\mathrm{RMSE}_{\text {loosely }}}\right) & \text { if } & \frac{\mathrm{RMSE}_{\text {losely }}}{\mathrm{RMSE}_{\text {closely }}}<1\end{array}\right.$

Hence, this expression yields positive values when the closely coupled approach outperforms the loosely coupled integration.

The average improvement of the position, velocity, acceleration, and orientation error is visualized in Fig. 5. It can be seen that the loosely coupled integration performs slightly better whenever the receiver is tracking more than four visible satellites ("all"). This difference may be due to differing filter settings (e.g. the processing noise). At the critical number of $4 \mathrm{SVs}$ in view, the performance of the closely coupled approach is marginally better (10-30\%). With further decrease of tracked SVs, the improvement provided by the closely coupled approach increases and becomes maximum for $3 \mathrm{SVs}$ and a GPS outage duration of $40 \mathrm{~s}$. The trajectory depicted in Fig. 6 illustrates this behavior: the closely coupled solution follows very well the reference track whereas the loosely coupled solution diverges considerably. For fewer than $3 \mathrm{SVs}$, the improvement provided by the closely coupled integration is reduced and the difference is again marginal for $1 \mathrm{SV}$ tracked.

We now look in more detail on the gain in position and orientation accuracy provided by the closely coupled integration scheme under partial satellite constellation.

Figure 7 shows the position errors for different duration of satellite tracking outages $(10,15,20$ and $30 \mathrm{~s})$. For outage times up to $15 \mathrm{~s}$, the difference in performance between both approaches is marginal. The vertical component presents a peak at four satellites which is less dominant in the closely coupled approach. In such situations where no redundant satellites are available, the filter probably overweights the remaining pseudoranges with 
Fig. 5 Accuracy improvement of the closely coupled integration approach with respect to the loosely coupled approach as a function of the number of SVs and the simulated outage duration

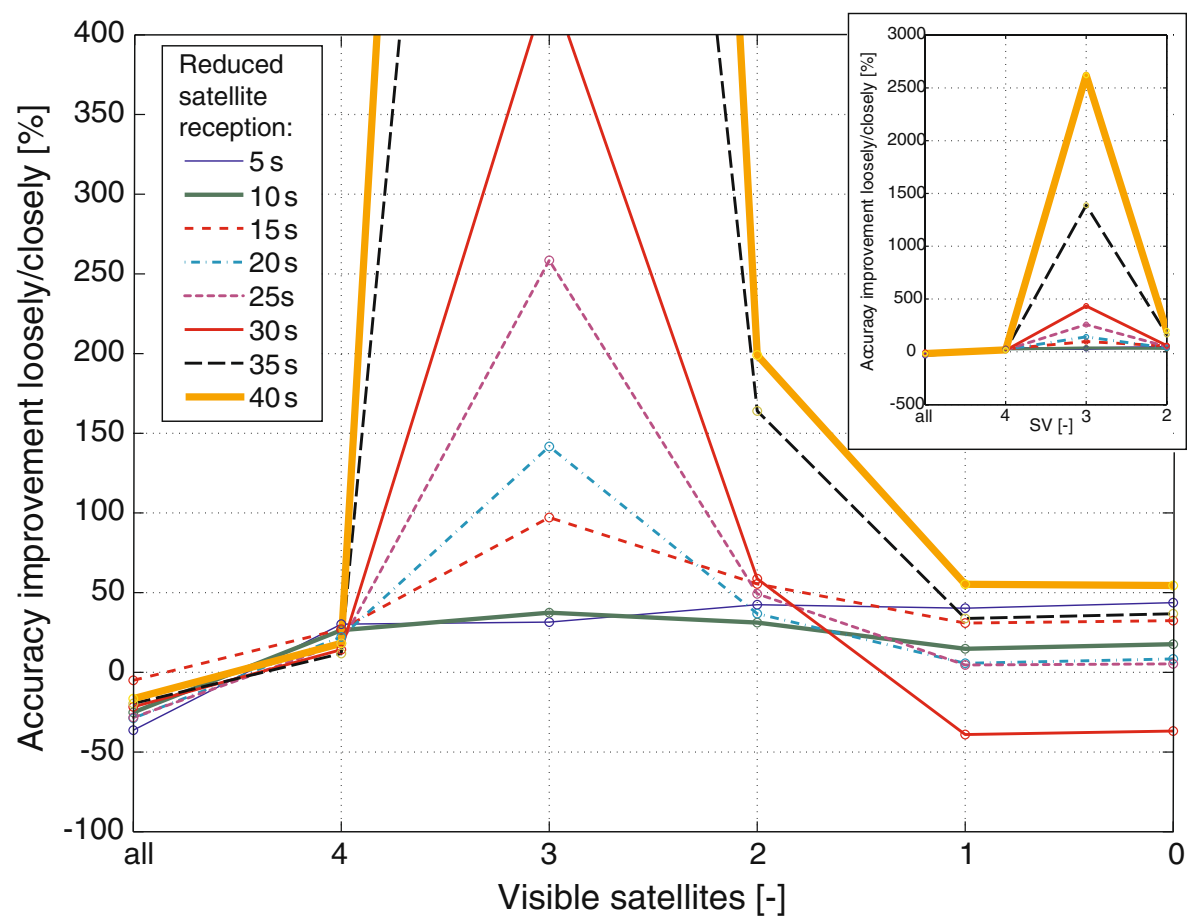

Fig. 6 Trajectory during a $40 \mathrm{~s}$ GPS outage computed with 3 SVs applying a closely and a loosely coupled integration approach

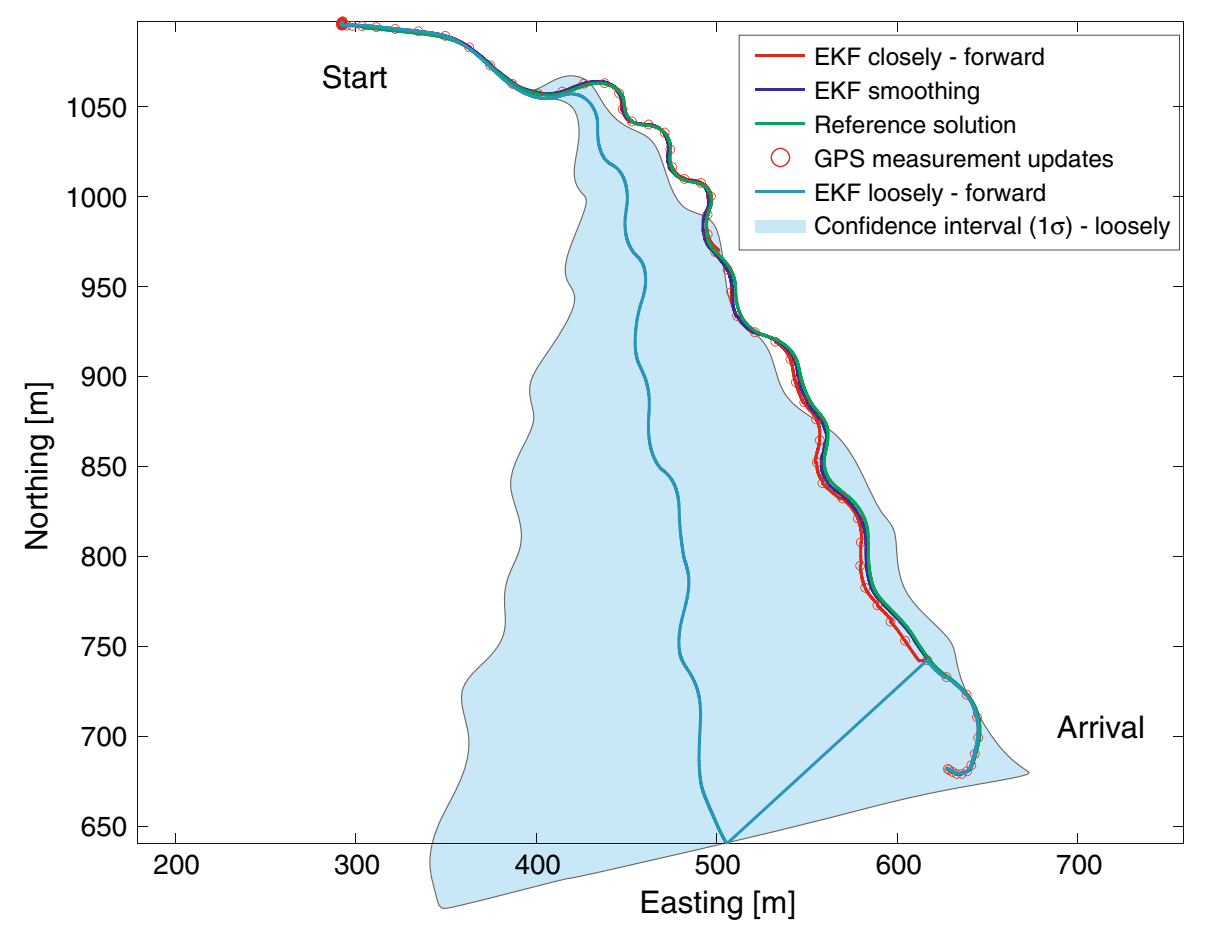

respect to the inertial solution. On the other hand, for outage times larger than $20 \mathrm{~s}$ the closely coupled approach outperforms the loosely coupled strategy, with a major improvement at $3 \mathrm{SVs}$. However, for less than three satellites and outage times larger than $30 \mathrm{~s}$, the position error increases rapidly in correspondence to MEMS-inertial sensor characteristics. During such scenarios, neither approach is appropriate to satisfy accuracy requirements in sports (Table 1).

The development of the orientation errors is slower in time. Hence, the difference between the integration approaches becomes apparent only for larger outages as depicted in Fig. 8. There, the closely coupled strategy performs better. With this approach, the orientation error 

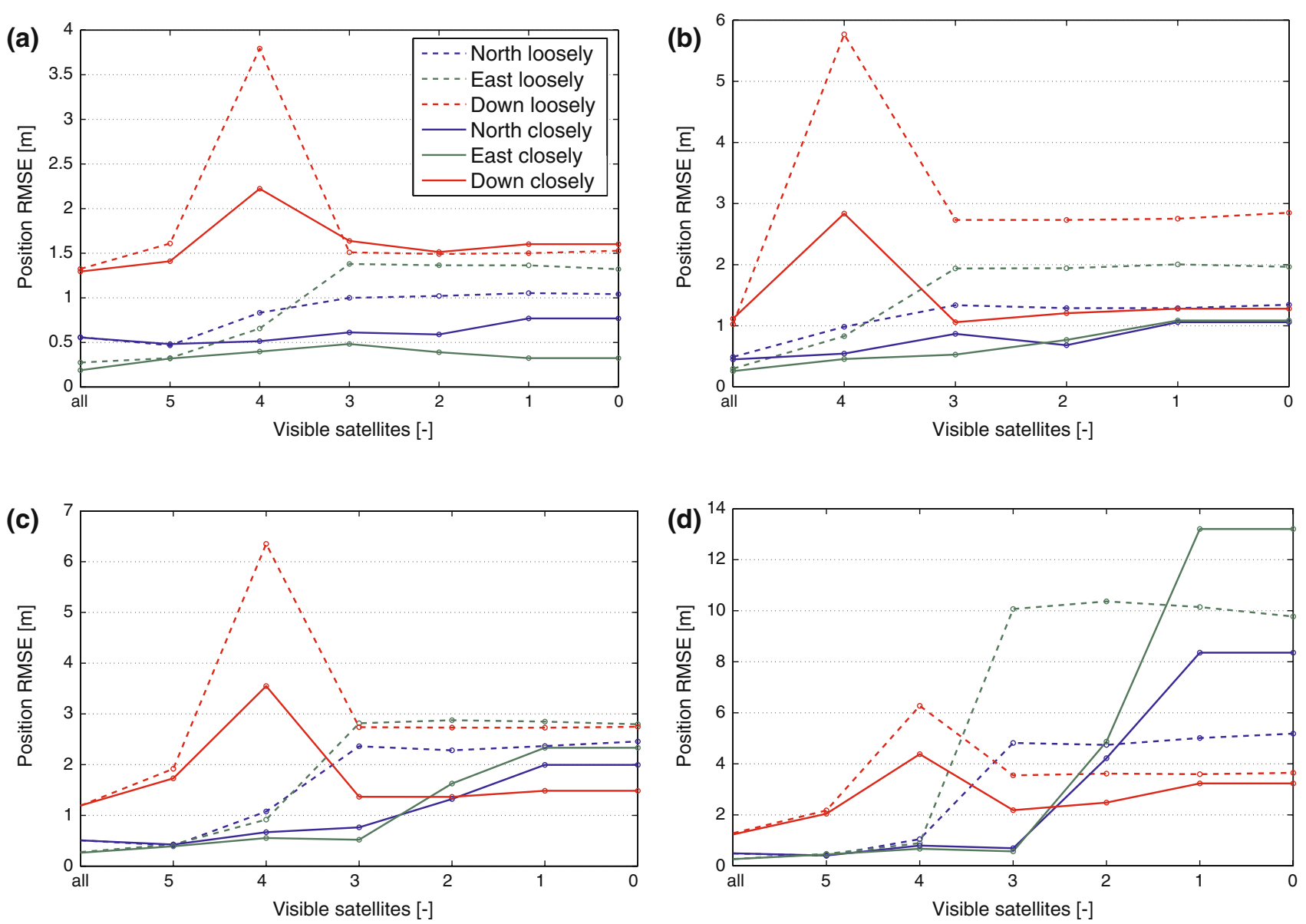

Fig. 7 Position error (RMS) during a data outage of $10 \mathrm{~s} \mathrm{(a),} 15 \mathrm{~s} \mathrm{(b),20} \mathrm{s} \mathrm{(c)} \mathrm{and} 30 \mathrm{~s}$ (d) as a function of the number of satellites
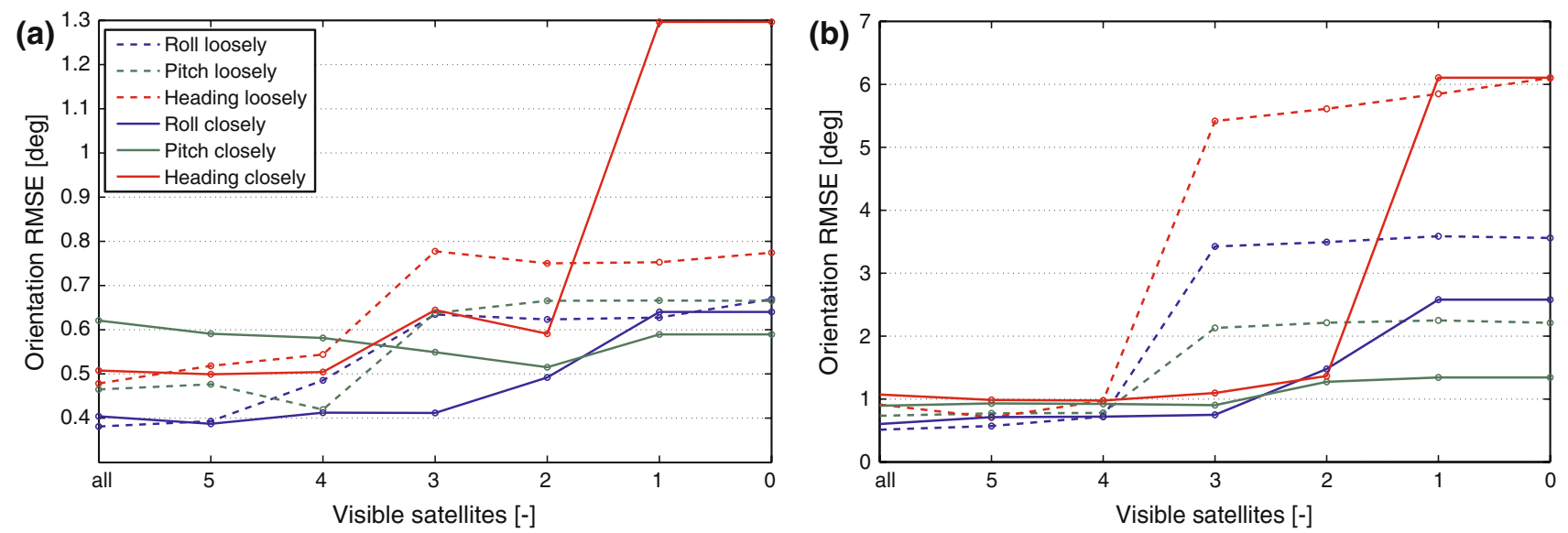

Fig. 8 Orientation error (RMS) during a data outage of $20 \mathrm{~s}$ (a) and $40 \mathrm{~s}$ (b) as a function of the number of satellites

remains bounded and satisfactory results are provided even for only $2 \mathrm{SVs}$.

We have seen that the loosely coupled strategy provides similar performance at full or partial satellite constellation (more than $3 \mathrm{SVs}$ ) which confirms the findings in (Schwarz et al. 1994). For $3 \mathrm{SVs}$ and outages larger than $15 \mathrm{~s}$ the closely coupled approach is certainly better but this difference becomes negligible at $2 \mathrm{SVs}$ and diminishes completely at $1 \mathrm{SV}$. This confirms the simulations presented by Wei and Schwarz (1990); Sukkarieh and George 
(2005). Therefore, the closely coupled integration can provide better results under certain circumstances.

However, the analyzed data corresponds to $1 \mathrm{~h}$ period during which the satellite geometry did not change significantly. Hence, no general conclusion for arbitrary satellite constellations is possible.

\section{Initialization performance assessment}

The initial alignment is a critical phase in GPS/MEMSIMU integration. Two methods for the initialization of the orientation were previously introduced.

Evaluation based on simulations

We will first investigate the sensitivity of both algorithms to sensor biases. Indeed, it is important to verify whether the orientation error caused by typical accelerometer biases and magnetic disturbances can be tolerated. For this purpose, a synthetic set of inertial and magnetic measurements was generated. The measurement biases were alternatively added to each axis (Fig. 9, 10). The accelerometer measurements were "fixed" at normal gravity and the magnetic measurements to the value of the Earth magnetic field at the position $\left(46^{\circ} \mathrm{N}, 7^{\circ} \mathrm{E}, 1,775 \mathrm{~m}\right)$ and epoch 2007 . Typical accelerometer biases encountered with the MEMS-type sensors amount to $0.2 \mathrm{~m} / \mathrm{s}^{2}$. Such biases cause maximum orientation errors of approximately $2 \mathrm{deg}$ (Fig. 9). On the other hand, soft magnetic disturbances and magnetometer biases have a much larger impact on the initial orientation. Indeed, these errors can range up $0.2 \mathrm{~T} / \mathrm{T}$ and cause orientation errors up to $20 \mathrm{deg}$ (Fig. 10). Experience shows, however, that such errors are rapidly mitigated by the GPS updates and do not generate numerical instabilities in the KF. Hard magnetic disturbances (i.e. sign flipping or complete overmasking) induce variable orientation errors up to 50 deg which may cause filter divergence. Such cases can be provoked by the vicinity of other electronic accessories (e.g. computers) or metallic components of the sport equipment (e.g. in motorsports). In some cases the absolute value of the sensor bias can be reduced by sensor precalibration (Waegli et al. 2007b).

\section{Experimental evaluation}

The limitations of both initialization algorithms with respect to the MEMS sensor error characteristics are also analyzed using the tactical-grade GPS/INS as reference. The errors of the modified coarse alignment and of the QUEST algorithm are illustrated in Fig. 11 for one run. As depicted in the example, both algorithms converge rapidly after the start as soon as the dynamics increases and the inertial biases decorrelate from the orientation estimate (Waegli and Skaloud 2007). The initial differences between the two initial orientation solutions $(<0.5 \mathrm{deg})$ are negligible and have no impact on the filter convergence.
Fig. 9 Initial orientation error as a function of the initial accelerometer bias

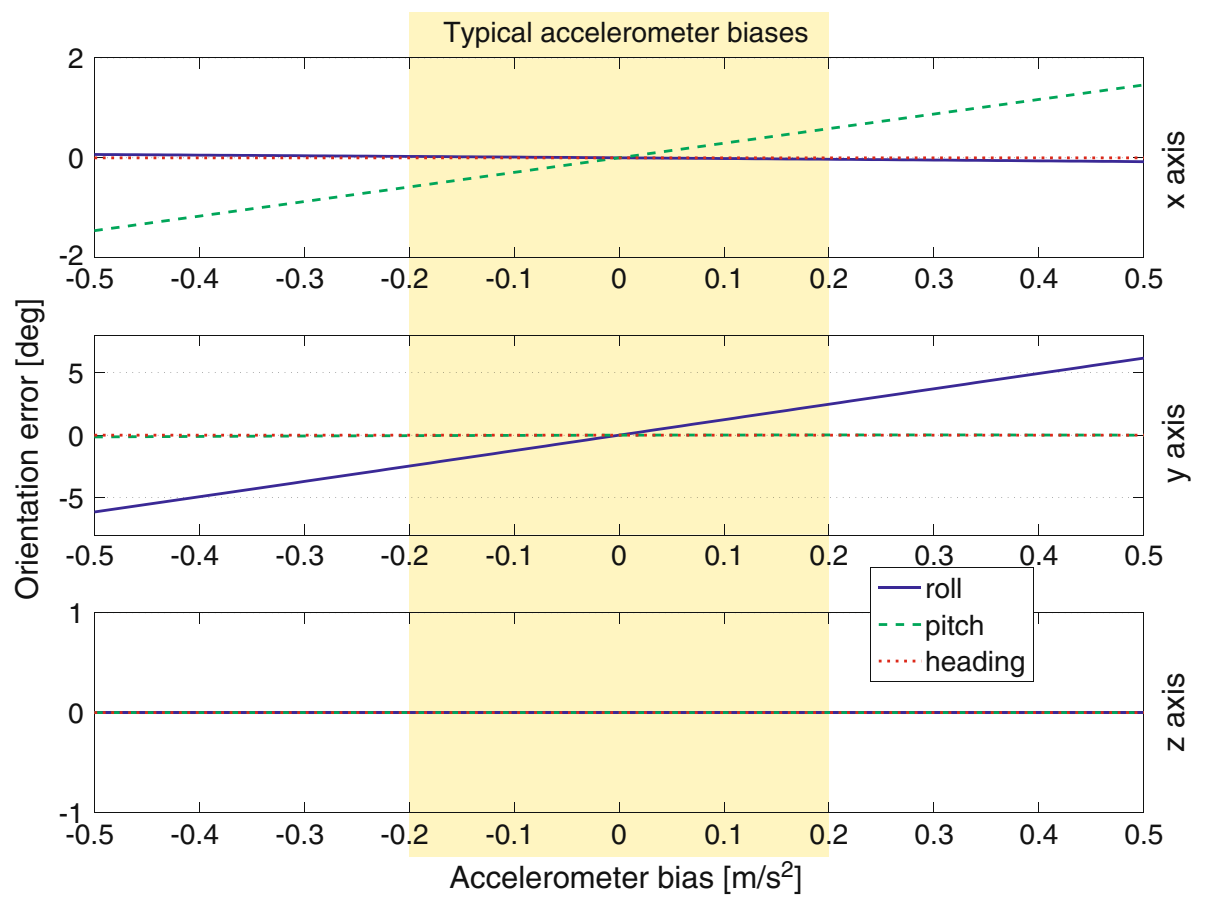


Fig. 10 Initial orientation error as a function of the initial magnetometer bias or magnetic disturbance

Fig. 11 Comparison of the coarse alignment and QUEST algorithm for the orientation initialization (run \#11)
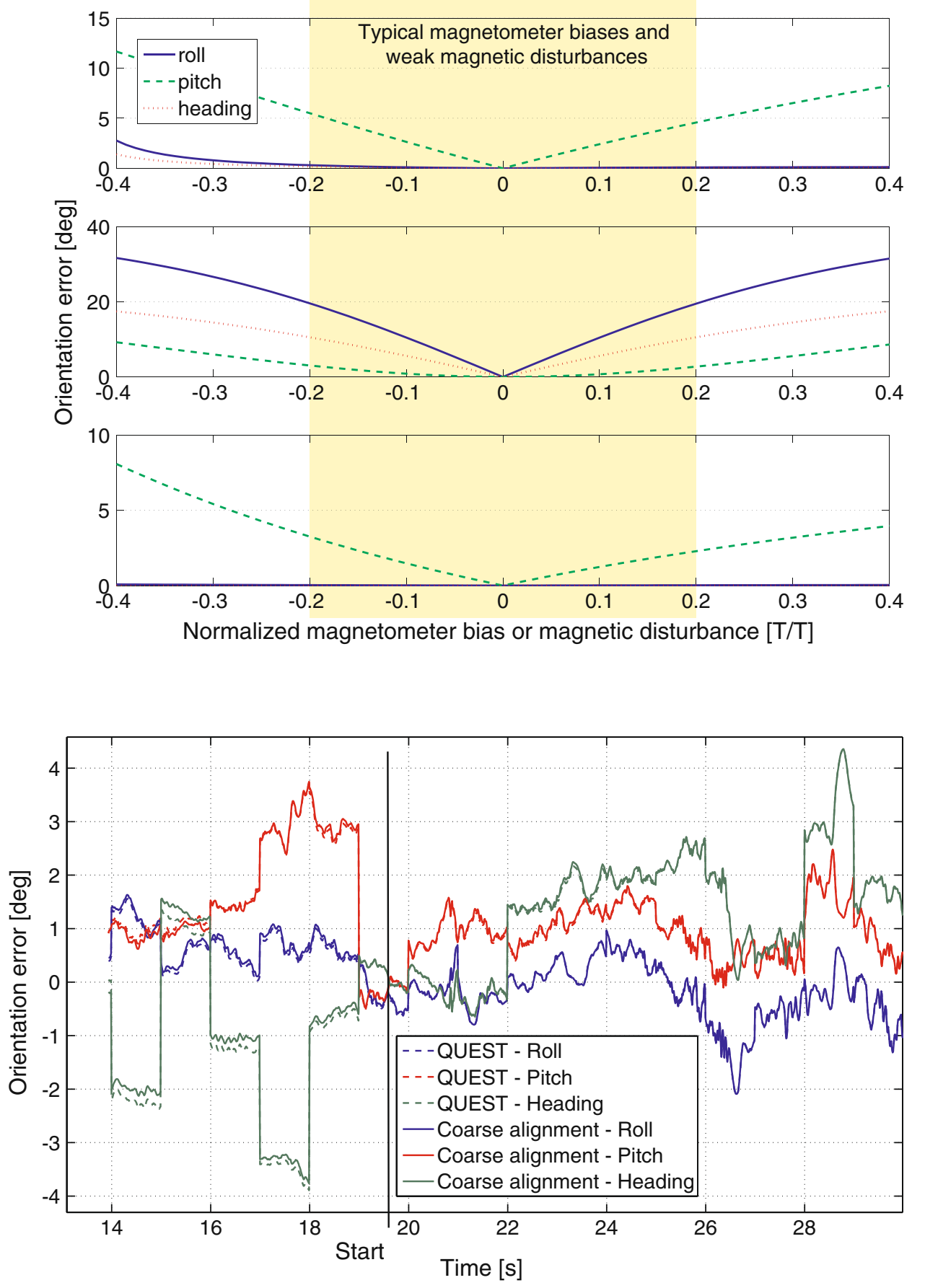

\section{Conclusion and perspectives}

In this article, we have investigated the potential of using L1-GPS/MEMS-IMU sensors for tracking sport performance. We presented and analyzed the merits of different integration strategies and initialization methods. The theoretical approaches were supported by simulations and experimental testing with the support of reference solutions.
From these investigations, the following conclusions are drawn:

- The loosely coupled integration strategy provides slightly increased performance over the closely coupled approach for full or partial (more than $3 \mathrm{SVs}$ ) satellite constellations, but this difference is marginal. For a constellation consisting of $3 \mathrm{SVs}$, the closely coupled approach clearly outperforms the loosely coupled 
method and allows maintaining a submeter accuracy up to $30 \mathrm{~s}$. In the orientation domain, good results can be achieved by the closely coupled integration even for only $2 \mathrm{SVs}$. On the contrary to tactical-grade IMUS, there is not a significant benefit for closely coupled integration using MEMS sensors when only $2 \mathrm{SVs}$ are available.

- For satellite outages longer than $20 \mathrm{~s}$ and less than $2 \mathrm{SVs}$ in view, the navigation errors become dominated by the inertial errors and exceed the accuracy requirements for sports application.

- The classical alignment methods used in GPS/INS are not applicable because of the sensor characteristics of the MEMS-IMU sensors. The first alternative for orientation initialization is a modified coarse alignment algorithm where the sensing of the Earth's rotation rate is replaced by the measurement of the Earth's magnetic field. The second approach is a quaternion estimation method, also based on accelerometer and magnetometer measurements. Both algorithms performed equally. However, the QUEST approach is more convenient for strapdown navigation using quaternions and guards automatically against singularity.

Acknowledgments This research is financed by TracEdge, based at Grenoble, France.

\section{References}

Bar-Itzhack IY (1996). REQUEST-a new recursive algorithm for attitude determination. In: Proceedings of the ION NTM, Santa Monica, CA, USA

Braasch MS (2001) Performance comparison of multipath mitigating receiver architectures. In: Proceedings of the IEEE aerospace conference, Big Sky, MT, USA

Bruton AM, Glennie CL, Schwarz KP (1999) Differentiation for high precision GPS velocity and acceleration determination. GPS Solut 2(4):4-21. doi:10.1007/PL00012771

Ge L, Han S, Rizos C (2000) Multipath mitigation of continuous GPS measurements using an adaptive filter. GPS Solut 4(2):19-30. doi:10.1007/PL00012838

Gelb A (1994) Applied optimal estimation. The MIT Press, Cambridge

Hatch R (1982). The synergism of gps code and carrier measurements. In: Proceedings of the 3rd international geodetic symposium on satellite Doppler positioning, Las Cruces, NM, USA

Hofmann-Wellenhof B, Lichtenegger H, Collins J (1992) GPS theory and practice. Springer, New York

How J, Pohlman N, Park CW (2002) GPS estimation algorithms for precise velocity, slip and race-track position measurements. In: Proceedings of the SAE motorsports engineering conference and exhibition

Li Y, Dempster A, Li B, Wang J, Rizos C (2006) A low-cost attitude heading reference system by combination of GPS and magnetometers and MEMS inertial sensors for mobile applications. J Glob Position Syst 5(1-2):90-97

Meditch JS (1969) Stochastic optimal linear estimation and control. McGraw-Hill, New York
Schuster MD, Oh SD (1981) Three-axis attitude determination from vector observations. J Guidance Contr 4(1):70-77. doi:10.2514/ 3.19717

Schwarz KP, Wei M, Van Gelderen M (1994). Aided versus embedded-a comparison of two approaches to gps/ins integration. In: Proceedings of the IEEE PLANS 1994, Las Vegas, NV, USA

Shin EH (2005) Estimation techniques for low-cost inertial navigation, UCGE report number 20219

Skaloud J, Limpach P (2003) Synergy of CP-DGPS, accelerometry and magnetic sensors for precise trajectography in ski racing. In: Proceedings of the ION GPS/GNSS 2003, Portland, OR, USA

Skaloud J, Merminod B (2000) DGPS-calibrated accelerometric system for dynamic sports events. In: Proceedings of the ION GPS, Salt Lake City, UT, USA

Sukkarieh S, George M (2005) Tightly coupled INS/GPS with bias estimation for UAV applications. In: Proceedings of the Australian conference on robotics and automation, Sydney, Australia

Titterton DH, Weston JL (1997) Strapdown inertial navigation technology, Peter Peregrinus Ltd

Törnqvist D (2006) Statistical fault detection with applications to IMU disturbances. Linköping, Sweden

Van Dierendonck AJ, Braasch MS (1997) Evaluation of GNSS receiver correlation processing techniques for multipath and noise mitigation. In: Proceedings of the ION NTM, Long Beach, CA, USA

Waegli A (2009) Trajectory determination and analysis in sports by satellite and inertial navigation. Ph.D. dissertation, EPFL, Lausanne, Switzerland

Waegli A, Skaloud J (2007) Assessment of GPS/MEMS-IMU integration performance in ski racing. In: Proceedings of ENCGNSS 2007 (TimeNav'07), Geneva, Switzerland

Waegli A, Meyer F, Ducret S, Skaloud J, Pesty R (2007a) Assessment of timing and performance based on trajectories from low-cost GPS/INS positioning. Sci Skiing IV:556-564

Waegli A, Skaloud J, Tomé P, Bonnaz JM (2007b) Assessment of the integration strategy between GPS and body-worn MEMS sensors with application to sports. In: Proceedings of the ION GNSS 2007, Fort Worth, TX, USA

Waegli A, Schorderet A, Prongué C, Skaloud J (2008) Accurate trajectory and orientation of a motorcycle derived from low-cost satellite and inertial measurement systems. Eng Sport 7:223230. doi:10.1007/978-2-287-09411-8_27

Wahba G (1965) A least squares estimate of spacecraft attitude. SIAM Rev 7(3):409. doi:10.1137/1007077

Wei M, Schwarz KP (1990) Testing a decentralized filter for GPS/ INS integration. In: Proceedings of the IEEE PLANS 1990, Las Vegas, NV, USA

Wendel J, Metzger J, Moenikes R, Maier A, Trommer GF (2005) A performance comparison of tightly coupled GPS/INS navigation systems based on extended and sigma point kalman filters. In: Proceedings of the ION GNSS, Long Beach, CA, USA

Yang Y, Hatch RR, Sharpe RT (2004) GPS multipath mitigation in measurement domain and its applications for high accuracy navigation. In: Proceedings of the ION GNSS 2004, Long Beach, CA, USA

Zhang K, Grenfell R, Deakin R, Li Y, Jason Z, Hahn A, Gore C, Rice $\mathrm{T}$ (2003) Towards a low-cost, high output rate, real-time gps rowing coaching and training system. In: Proceedings of the ION GPS/GNSS 2003, Portland, OR, USA

Zhang K, Deakin R, Grenfell R, Li Y, Zhang J, Cameron WN, Silcock DM (2004) GNSS for sports-sailing and rowing perspectives. J Glob Position Syst 3(1-2):280-289 